\title{
ПРОВЕДЕНИЕ КОНТРОЛЯ ЗНАНИЙ СТУДЕНТОВ С ПРИМЕНЕНИЕМ ДИСТАНЦИОННЫХ ОБРАЗОВАТЕЛЬНЫХ ТЕХНОЛОГИЙ ПО ПРОГРАММАМ ВЫСШЕГО ОБРАЗОВАНИЯ В СФЕРЕ ФИЗИЧЕСКОЙ КУЛЬТУРЫ И СПОРТА С УЧЕТОМ ТРЕБОВАНИЙ ПРОФЕССИОНАЛЬНЫХ СТАНДАРТОВ
}

\section{DEVELOPMENT OF A METHODOLOGY FOR MONITORING STUDENTS' KNOWLEDGE USING DISTANCE EDUCATIONAL TECHNOLOGIES FOR HIGHER EDUCATION PROGRAMS IN THE FIELD OF PHYSICAL CULTURE AND SPORTS, TAKING INTO ACCOUNT THE REQUIREMENTS OF PROFESSIONAL STANDARDS}

\section{P. Bordovskiy \\ L. Kiryanova \\ V. Zhukova \\ E. Shutova}

Summary: Improving education is the main driving force in the development and strengthening of any state. In the Russian Federation, issues of improving the educational process are a priority and are resolved at the level of ministries and departments. To address this issue in the field of physical culture and sports, there is a list of research topics, including those on education in the field of physical culture and sports, approved at the level of the Ministry of Sports of the Russian Federation. This article examines the main issues on the development of a system for diagnostics and monitoring of the educational outcomes according to the new educational standard of higher education $3++$. Also results of the testing using the developed system according to the programs of professional standards in the discipline "Information technologies in physical culture and sports (PhCandS) " were analyzed.

The developed system for monitoring the educational outcomes was developed using distance educational technologies and implemented on the official website of distance education NSU. P.F. Lesgaft, St. Petersburg.

Keywords: open education, education in the field of physical culture (FC), control and diagnostics, professional function, professional action, distance educational technologies, the formation of assessment tools.

\section{Введение}

оявление новых образовательных стандартов для по направлению подготовки Физическая культура происходит каждые несколько лет. Разработка но-
Бордовский Павел Георгиевич

К.п.н., дочент, НГУ им. П.Ф. Лесгафта (Санкт-Петербург) pbord@bk.ru

Кирьянова Людмила Александровна

К.п.н., доцент, Северо-Западный институт управления РАНХИГС, Санкт-Петербург

Жукова Валентина Ивановна

доцент, Уральский Федеральный Университет имени первого Президента России Б.Н. Ельцина, Екатеринбург

Шутова Евгения Борисовна

старший преподаватель, Уральский Федеральный

Университет имени первого Президента России Б.Н.

Ельчина, Екатеринбург

Аннотация: Совершенствование образования является основной движущей силой в развитии и укреплении любого государства. В Российской Федерации вопросы совершенствования образовательного процесса являются приоритетными и решаются на уровне министерств и ведомств. Для решения этого вопроса в области физической культуры и спорта существует перечень тем научных исследований, в том числе и по вопросам образования в сфере ФКиС, утверждённый на уровне Министерства спорта Российской Федерации. В этой работе рассматриваются основные вопросы по разработке системы диагностики и контроля результатов образовательного процесса по новому образовательному стандарту высшего образования $3++$, а, так же выполнен анализ результатов проведённого тестирования с использованием разработанной системы по программам профессиональных стандартов по дисциплине «Информационные технологии в физической культуре и спорте (ФКиС)».

Разработанная система контроля результатов образовательного процесса была разработана с применением дистанционных образовательных технологий реализована на официальном сайте дистанционного обучения НГУ им. П.Ф. Лесгафта, Санкт-Петербург.

Ключевые слова: открытое образование, образование в сфере физической культуры (ФК), контроль и диагностика, трудовая функция, трудовое действие, дистанционные образовательные технологии, формирование оценочных средств.

вых стандартов связана с постоянно повышающимися требованиями к подготовке специалистов в области физической культуры и спорта. Последний образовательный стандарт был утвержден 19.09.2017 приказом Министерства образования и науки Российской Федерации [3] 
Реализация данного образовательного стандарта производится уже в течение четырех лет и понимание эффективности обучения студентов по этому стандарту является весьма важным и актуальным вопросом. в НГУ им. П.Ф. Лесгафта, Санкт-Петербург помимо традиционной формы обучения создано и активно применяется обучение с применением дистанционных образовательных технологий. В связи с этим второй задачей исследования мы рассматривали вопросы эффективности обучения студентов по этому стандарту с применения дистанционного дистанционных образовательных технологий и реализованных на сайте ДО НГУ им. П.Ф. Лесгафта, Санкт-Петербург [1]. Данные технологии имеют довольно много преимуществ в вопросах проведения массовых и часто используемых контролей [2]. Дистанционная обучение студентов в нашем университете основано на одной из лучших в мире дистанционной платформе - Moodle. Данная платформа имеет довольно мощные механизмы проверки качества усвоения знаний студентами в процессе обучения, что позволяет проводить исследования такого типа [5].

\section{Шель исследования}

Проверить эффективность реализации нового государственного образовательного стандарта высшего образования - бакалавриат по направлению подготовки 49.03.01 Физическая культура, как при традиционной форме обучения, так и с применением дистанционных образовательных технологий, применение которых в связи с приказом Минобрнауки России от 11.11.2020 N 1402 стало обязательным [4].

Для определения сферы проведения контролей был выполнен анализ всех обобщённых трудовых функций, трудовых функций, трудовых действий, необходимых умений и необходимых знаний который показал, что все требования, предъявляемые к результатам обучения на 5 и 6 уровнях можно объединить в следующие относительно самостоятельные разделы изучаемы в дисциплине «Информационные технологии в ФКиС»

Хочется отметить, что есть ещё довольно большое количество трудовых функций, необходимых знаний и умений, в которых требуется довольно высокий уровень владения средствами вычислительной техники и пакетами прикладных программ, но который официально не относится к дисциплине «Информационные технологии в ФКиС».

Кроме этого, есть довольно большое количество специальных программами по виду спорта в т.ч., программы проведения соревнований, планирования, контроля физических, психических, физиологических и прочих характеристик и прочее (седьмая группа по представленной выше таблице), которые не могут изучаться в дис- циплине «Информационные технологии в ФКиС» так как не относятся к общим программам, а являются узкоспециализированными для конкретного вида деятельности (спорта, специализации, подразделения и прочее). Такие программы должны изучаться непосредственно в разделах ТИМИС по выбранной специальности [6].

Следующим этапом стало формирование оценочных средств для промежуточной аттестации в форме тестовых заданий по программам бакалавриата и магистратуры.

Формирование оценочных средств проводилось с учётом специфики различных трудовых функций, а также, с учётом проверки не только необходимых знаний, но и умений и опыта применения.

При составлении тестов применялись вопросы следующих типов:

1. Множественный выбор, позволяющий студенту выбрать правильный ответ (или ответы) из предложенных вариантов.

2. Краткий ответ, предлагающий студенту написать правильный ответ самостоятельно.

3. На соответствие, предлагающий студенту сопоставить несколько вариантов вопросов с несколькими вариантами ответов

4. Эсcе

При формировании банка вопросов разного типа существуют различные плюсы и минусы для каждого типа вопросов.

Вопросы первого типа - множественный выбор имеют преимущество в том, что позволяют довольно развёрнуто и подробно описать суть вопроса и варианты ответов, что позволяет формировать оценочные средства практически по всем трудовым функциям и различной степени сложности вопроса. Однако данная категория имеет и довольно большое количество минусов, которые заключаются в возможности простого угадывания правильного ответа или в логическом анализе и исключении альтернативных вариантов ответов. И фактически происходит процесс узнавания материала, а не знание конкретного ответа, т.е. в случае необходимости воспроизвести ответ студент этого сделать не сможет. Кроме этого, зачастую возникают сложности с созданием альтернативных вариантов ответов, так как углублённое знание и формулировка ответов зачастую исключают варианты вариативности. Однако данный тип вопросов можно широко использовать в таких разделах трудовых функций, как состояние вопроса, история развития, современное состояние и прочие общие темы.

Тестовые вопросы с кратким ответом на наш взгляд являются более актуальными, так как позволяют до- 
Таблица 1.

\begin{tabular}{|c|c|c|}
\hline & Разделы дисциплины ИТ в ФКиС & Основные трудовые действия, необходимые умения и необходимые знания \\
\hline 1 & $\begin{array}{l}\text { Основы работы на ПК, управление созданием, хранени- } \\
\text { ем и передачей информации }\end{array}$ & Основы работы с персональным компьютером, с электронной почтой и браузерами \\
\hline 2 & Владение текстовым редактором & $\begin{array}{l}\text { Оценка эффективности подготовки обучающихся с использованием современных инфор- } \\
\text { мационных и компьютерных технологий, в том числе текстовых редакторов и электрон- } \\
\text { ных таблиц, в своей деятельности; } \\
\text { Вести первичную учетно-отчетную документацию по реализации программ спортивной } \\
\text { подготовки в электронном и бумажном виде }\end{array}$ \\
\hline 3 & $\begin{array}{l}\text { Владение табличным процессором (электронными та- } \\
\text { блицами) }\end{array}$ & $\begin{array}{l}\text { Оценка эффективности подготовки обучающихся с использованием современных инфор- } \\
\text { мационных и компьютерных технологий, в том числе текстовых редакторов и электрон- } \\
\text { ных таблиц, в своей деятельности; } \\
\text { Вести первичную учетно-отчетную документацию по реализации программ спортивной } \\
\text { подготовки в электронном и бумажном виде; } \\
\text { Систематический учет, анализ, обобщение результатов работы, в том числе с использова- } \\
\text { нием электронных форм }\end{array}$ \\
\hline 4 & Владение презентационными программами & $\begin{array}{l}\text { Использовать информационные технологии, в том числе средства подготовки и демон- } \\
\text { страции презентаций, видеофильмов, иных образовательных ресурсов }\end{array}$ \\
\hline 5 & $\begin{array}{l}\text { Владение мультимедийными программами (обработка } \\
\text { аудио и видео информации) }\end{array}$ & $\begin{array}{l}\text { Использовать информационные технологии, в том числе средства подготовки и демон- } \\
\text { страции презентаций, видеофильмов, иных образовательных ресурсов; } \\
\text { Выполнение с обучающимися анализа соревновательной деятельности в виде спорта пу- } \\
\text { тем изучения кино- и видеоматериалов специальной литературы }\end{array}$ \\
\hline 6 & $\begin{array}{l}\text { Владение программами интернет общения (браузеры, } \\
\text { почта, соцсети) }\end{array}$ & $\begin{array}{l}\text { Основы работы с персональным компьютером, с электронной почтой и браузерами; } \\
\text { Пользоваться информационно-коммуникационными технологиями и средствами связи }\end{array}$ \\
\hline 7 & $\begin{array}{l}\text { Владение специальными программами по виду спорта } \\
\text { В т.ч. Программами проведения соревнований, плани- } \\
\text { рования, контроля физических, психических, физиоло- } \\
\text { гических и прочих характеристик. Программами пла- } \\
\text { нирования и учёта спортивной деятельности и прочее. }\end{array}$ & $\begin{array}{l}\text { Оценка эффективности подготовки обучающихся с использованием современных инфор- } \\
\text { мационных и компьютерных технологий, в том числе текстовых редакторов и электрон- } \\
\text { ных таблиц, в своей деятельности; } \\
\text { Методики контроля и оценки физической подготовленности спортсменов; } \\
\text { Определять справедливость судейства; } \\
\text { Выполнение со спортсменами анализа собственной соревновательной практики, изучения } \\
\text { кино- и видеоматериалов специальной литературы, в том числе иностранной }\end{array}$ \\
\hline
\end{tabular}

вольно точно определить уровень необходимых знаний у студентов. Однако у вопросов данного типа тоже есть недостатки, они не позволяют оценивать развёрнутые ответы студентов и ввести градацию ответов. Основной плюс данного варианта, он показывает конкретно что знает студент.

Вопросы на соответствие удобны в том случае, если оценить нужно какой-то перечень знаний или действий, имеющих определённые связи или порядок следования. Причём количество вопросов и ответов могут не совпадать, что позволяет оценивать различные формулировки ответов студентов. Однако, данные вопросы являются довольно сложными и громоздкими для формирования банка вопросов.

Очень интересными для формирование оценочных средств промежуточной аттестации в форме тестовых заданий являются вопросы типа эссе. Такая группа вопросов стала наиболее популярна в большинстве видов тестирования, так как позволяет создавать сложные вопросы, предполагающие развёрнутые творческие ответы. Вопросы данного типа могут широко использоваться для проверки необходимых умений и в какой-то степени приобретённого опыта обучаемого. В вопросе такого типа можно совершенно просто поставить перед студентом задачу, требующую творческого решения, с указанием на собственный опыт решения задачи такого типа. Основной недостаток вопросов такого типа, это то, что они в отличие от предыдущих трёх типов вопросов не могут быть оценены автоматически и требуют ручного оценивания. Вопросы типа эссе на наш взгляд должны более широко использоваться для программ магистратуры.

При формировании оценочных средств промежуточной аттестации в форме тестовых заданий по программам бакалавриата и магистратуры мы использовали все четыре типа вопросов. Вопросы с множественным выбором используются для оценки самого низшего уровня знаний и даже $100 \%$ правильные ответы на эти вопросы не могут быть оценены на «отлично». Вопросы с кратким 
ответом и на соответствие при высоком проценте правильных ответов позволяют с высокой вероятностью оценить уровень необходимых знаний студента и может быть поставлена оценка «отлично».

На наш взгляд, первых трёх групп вопросов может быть достаточным для оценочных средств промежуточной аттестации для 5-го уровня.

Для оценки полученных умений и опыта применения мы рекомендуем использовать вопросы типа эссе с элементами сравнительного анализа или описанием собственного опыта работы и рекомендуется для 6 и 7 уровня.

Практика формирования оценочных средств промежуточной аттестации в форме тестовых заданий по программам бакалавриата и магистратуры показала, что для объективного применения тестовых заданий необходимо исключить повторяемость вопросов при неоднократном тестировании, так как это приводит к простому заучиванию конкретных ответов на вопросы. Рекомендуется иметь или набор из нескольких вариантов аналогичных тестов и варианты не должны повторяться или же иметь большую базу (банк) вопросов для каждого те- ста как минимум в 3-4 раза превосходящую количество вопросов, представляемых студенту.

Существует ещё одна проблема при формирования оценочных средств, она заключается в довольно большой зависимости оценочных средств от конкретных учебно-методических материалов, которые используются при обучении, а в настоящее время, когда наличие сети интернет позволяет использовать огромное количество информационных источников, в том числе и не всегда соответствующих предъявляемым к обучению требованиям, как в сторону усложнения и углубления изучаемого материала, так и в сторону популистской некорректной информации. Таким образом, рекомендуется для подготовки к тестированию обязательно указывать студентам тот перечень учебно-методических материалов, который позволят корректно формулировать и трактовать вопросы и ответы оценочных средств.

По каждому разделу дисциплины были созданы группы тестовых вопросов и по ним было проведено тестирование.

Результаты тестирования представлены в таблице 2.

\begin{tabular}{|c|c|c|c|c|c|}
\hline № раздела & Название раздела & $\begin{array}{l}\text { Средний балл } \\
(\max =10)\end{array}$ & $\begin{array}{l}\text { Удовл и выше } \\
-\%\end{array}$ & $\begin{array}{c}\text { Хорошо и выше } \\
-\%\end{array}$ & $\begin{array}{c}\text { Отлично } \\
-\%\end{array}$ \\
\hline 1 & $\begin{array}{l}\text { Основы работы на ПК, управление созданием, хранением } \\
\text { и передачей информации }\end{array}$ & 6,6 & 63,0 & 51,0 & 25,0 \\
\hline 2 & Владение текстовым редактором & 3,6 & 47,0 & 33,0 & 6,0 \\
\hline 3 & Владение табличным процессором (электронными таблицами) & 3,5 & 42,0 & 25,0 & 22,0 \\
\hline 4 & Владение презентационными программами & 8,6 & 100,0 & 88,0 & 83,0 \\
\hline 5 & $\begin{array}{l}\text { Владение мультимедийными программами (обработка аудио } \\
\text { и видео информации) }\end{array}$ & 4,8 & 54,0 & 31,0 & 18,0 \\
\hline 6 & $\begin{array}{l}\text { Владение программами интернет общения (браузеры, почта, } \\
\text { соцсети) }\end{array}$ & 8,8 & 100,0 & 85,0 & 74,0 \\
\hline
\end{tabular}

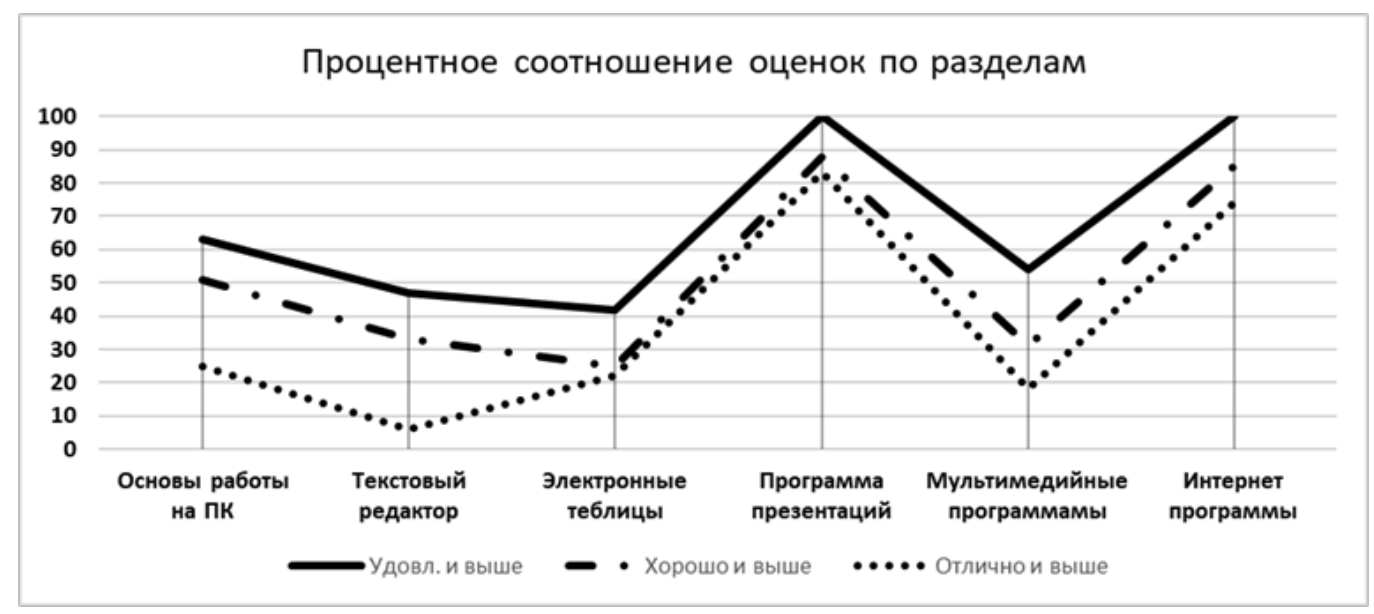

Рис. 1. Процентное соотношение оценок по разделам. 
На графике отображены процентные соотношения оценок «Удовлетворительно», «Хорошо» и «Отлично» у студентов по перечисленным в таблице разделам.

Анализ результатов проведенного тестирования показал, что уровень знаний студентов по основным разделам дисциплины в целом соответствуют требованиям, предъявляемым к результатам обучения для 5 и 6 уровня образовательного стандарта. Кроме этого, из графика можно увидеть, что знания по текстовым редакторам, с которыми чаще всего сталкиваются рядовые пользователи, оказались ниже ожидаемого уровня, особенно для оценки «Отлично» и выше. Самые высокие знания студенты ожидаемо показали по группам программ работы с презентациями и в сети интернет. Остальные категории знаний находятся на довольно хорошем среднем уровне.

\section{Выводы}

1. Проведение контроля знаний студентов с применением дистанционных образовательных технологий на основе тестирования является удобным и довольно эффективным инструментом в образовательном процессе и может быть рекомендован к применению в высших учебных заведениях, практически по всем дисциплинам и для всех видов контроля, включая промежуточные контроли.

2. Проведенное исследование показало, что обучение студентов по новому профессиональному образовательному стандарту 3++ позволяет достигнуть высоких результатов в знаниях, умениях и полученном опыте по основным разделам исследуемой дисциплины «Информационные технологии в ФКиС»

\title{
ЛИТЕРАТУРА
}

1. ДО НГУ им. П.Ф. Лесгафта, Санкт-Петербург, [Электронный ресурс] // URL: http://do.lesgaft.spb.ru/ course/index.php?categoryid=9 (дата обращения: 13.03.2021).

2. Олейник, Е.А., Ткачук М.Г., Бордовский П.Г. Современные педагогические аспекты дистанционных технологий в образовательном процессе в физкультурном вузе // Ученые записки университета имени П.Ф. Лесгафта. - 2019. - Т. 171. - № 5. - С. 220-223.

3. Приказ Минобрнауки РФ от 19 сентября 2017г. № 940 «0б утверждении федерального государственного образовательного стандарта высшего образования - бакалавриат по направлению подготовки 49.03.01 Физическая культура». [Электронный ресурc] - URL: https://rg.ru/2017/10/18/minobr-prikaz940site-dok.html/

4. Приказ Минобрнауки России от 11.11.2020 N 1402 "0 мерах по снижению рисков распространения новой коронавирусной инфекции в образовательных организациях высшего образования." [Электронный ресурc]/URL: - http://www.consultant.ru/document/cons_doc_LAW_367473 (дата обращения 06.03.2021)

5. Филиппов С.С., Закревская Н.Г., Бордовский П.Г. Исследование готовности преподавателей к деятельности в электронной информационно-образовательной среде университета / Национальный государственный университет физической культуры, спорта и здоровья им. П.Ф. Лесгафта, Санкт-Петербург // Уч. записки университета им. П.Ф. Лесгафта. - 2016. - № 8 (138). - С. 234-240.

6. LMS Data - Spring 2020 Updates. Edutechnica. [Electronic resource]. URL: - https://edutechnica.com/2020/02/02/Ims-data-spring-2020-updates/ (accessed: 02/02/2020).

\author{
с Бордовский Павел Георгиевич (pbord@bk.ru), Кирьянова Людмила Александровна, \\ Жукова Валентина Ивановна, Шутова Евгения Борисовна. \\ Журнал «Современная наука: актуальные проблемы теории и практики»
}

